\title{
INEQUALITIES FOR PERMANENTS AND PERMANENTAL MINORS OF ROW SUBSTOCHASTIC MATRICES*
}

\author{
ZHI CHEN ${ }^{\dagger}$, JIAWEI LI ${ }^{\ddagger}$, LIZHEN YANG ${ }^{\ddagger}$, ZELIN ZHU ${ }^{\ddagger}$, AND LEI CAO $§$
}

\begin{abstract}
In this paper, some inequalities for permanents and permanental minors of row substochastic matrices are proved. The convexity of the permanent function on the interval between the identity matrix and an arbitrary row substochastic matrix is also proved. In addition, a conjecture about the permanent and permanental minors of square row substochastic matrices with fixed row and column sums is formulated.
\end{abstract}

Key words. Row substochastic matrices, Doubly stochastic matrices, Doubly substochastic matrices, Transportation polytopes, Permanents.

AMS subject classifications. 15A $15,15 \mathrm{~A} 69$.

1. Introduction. Let $R=\left(r_{1}, \ldots, r_{m}\right)$ and $S=\left(s_{1}, \ldots, s_{n}\right)$ be nonnegative vectors satisfying

$$
\sigma=\sum_{i=1}^{m} r_{i}=\sum_{j=1}^{n} s_{j}
$$

The transportation polytope $\mathcal{U}(R, S)$ is the set of all $m \times n$ nonnegative matrices with row sum vector $R$ and column sum vector $S$, where $r_{i}$ and $s_{j}$ denote the $i$ th row sum and the $j$ th column sum, respectively. The matrices in $\mathcal{U}(R, S)$ are called transportation matrices. The polytope $\mathcal{U}(R, S)$ is non-empty if and only if equation (1.1) holds. By specializing $R=S=(1, \ldots, 1) \in \mathbb{R}^{n}$ in $\mathcal{U}(R, S)$, we obtain the convex polytope $\Omega_{n}$ of all $n \times n$ doubly stochastic matrices. The extreme points of $\Omega_{n}$, characterized by Birkhoff [1], are all $n \times n$ permutation matrices. Mirsky [7] investigated $\omega_{n}$, the convex polytope of all $n \times n$ doubly substochastic matrices, which are nonnegative matrices whose row and column sums are at most 1 , and showed that the extreme points of $\omega_{n}$ are all $n \times n$ subpermutation matrices. An $n \times n$ nonnegative matrix is said to be row stochastic if each row sum is equal to one. If each row sum is allowed to be less than or equal to one, then it is said to be a row substochastic matrix. All $n \times n$ row (sub)stochastic matrices form a convex polytope.

Let $A=\left[a_{i, j}\right]$ be an $n \times n$ matrix and $S_{n}$ be the symmetric group of order $n$. The permanent of $A$ is the scalar-valued function of $A$ defined by

$$
\operatorname{per}(\mathrm{A})=\sum_{\pi \in S_{n}} a_{1 \pi(1)} a_{2 \pi(2)} \cdots a_{n \pi(n)},
$$

where the summation extends over all $n$ ! permutations in $S_{n}$. Some inequalities involving diagonal sums, permanents and matrices in $\omega_{n}$ have been investigated in $[3,4,5]$. Denote $A_{i, j}$ the $(n-1) \times(n-1)$ matrix

\footnotetext{
*Received by the editors on July 16, 2019. Accepted for publication on November 15, 2019. Handling Editor: Raphael Loewy. Corresponding Author: Zhi Chen.

$\dagger$ Department of Mathematics, Nanjing Agricultural University, Jiangsu 210095, China (chenzhi@njau.edu.cn). Supported by the National Natural Science Foundation of China (no. 11601233), the Fundamental Research Funds for the Central Universities (no. KJQN201718), and the Natural Science Foundation of Jiangsu Province (no. BK20160708).

${ }^{\ddagger}$ Department of Mathematics, Nanjing Agricultural University, Jiangsu 210095, China. Lizhen Yang, Zelin Zhu, and Jiawei Li were supported by the Student Research Training Program (no. 1923A09).

$\S$ Department of Mathematics, Halmos College, Nova Southeastern University, FL, 33314, USA.
} 
obtained by deleting the $i$ th row and $j$ th column of $A$. In [2], Brualdi and Newman proved the following results.

Lemma 1.1. [2] Let $A \in \Omega_{n}$. Then

$$
\sum_{i=1}^{n}\left(1-a_{i, i}\right) \operatorname{per}\left(A_{i, i}\right) \leq 1-\operatorname{per}(A)
$$

Theorem 1.2. [2] Let $A \in \Omega_{n}, 0 \leq \alpha \leq 1$. Then

$$
\operatorname{per}(\alpha I+(1-\alpha) A) \leq \alpha+(1-\alpha) \operatorname{per}(A)
$$

In this paper, we prove some inequalities involving permanents and permanental minors of row substochastic matrices which are generalizations of Lemma 1.1 and Theorem 1.2 by Brualdi and Newman. In addition, we formulate a conjecture regarding the minimum of a permanental function on square row substochastic matrices with fixed row and column sums.

2. The inequalities for permanents of doubly substochastic matrices. Throughout this paper, we confine ourselves to square matrices. We write $A \geq 0$ for a nonnegative matrix $A$ and denote the trace of a square matrix $A$ by $\operatorname{tr}(A)$.

Lemma 2.1. Let $A$ be a square matrix of order $n$ and $A \geq 0$. If $0 \leq a_{i, i} \leq 1$ for all $1 \leq i \leq n$, then

$$
\operatorname{tr}(A) \leq n-1+\operatorname{per}(A)
$$

If $A$ is a diagonal matrix, then the inequality holds with equality if and only if at least $n-1$ diagonal elements of $A$ are equal to 1.

Proof. We first prove the following inequality.

$$
\operatorname{tr}(A)=a_{1,1}+\cdots+a_{n, n} \leq n-1+a_{1,1} a_{2,2} \cdots a_{n, n} .
$$

It is clear that (2.3) holds for $n=1$. Suppose that (2.3) holds when $n=k$, that is,

$$
a_{1,1}+\cdots+a_{k, k} \leq k-1+a_{1,1} a_{2,2} \cdots a_{k, k} .
$$

Adding $a_{k+1, k+1}$ to both sides of equation (2.4), we have

$$
a_{1,1}+\cdots+a_{k, k}+a_{k+1, k+1} \leq k-1+a_{1,1} a_{2,2} \cdots a_{k, k}+a_{k+1, k+1} .
$$

Since $0 \leq a_{i, i} \leq 1$ for all $1 \leq i \leq n$,

$$
k-1+a_{1,1} a_{2,2} \cdots a_{k, k}+a_{k+1, k+1} \leq k+a_{1,1} a_{2,2} \cdots a_{k+1, k+1} .
$$

Combining (2.5) and (2.6), we obtain (2.3). Since $A \geq 0$,

$$
\operatorname{per}(A) \geq a_{11} a_{22} \cdots a_{n n}
$$

and hence, (2.2) holds. Clearly, (2.7) holds with equality when $A$ is a diagonal matrix. 
If $A$ is a diagonal matrix with at most one element $a_{s, s}$ not equal to 1 , then

$$
\begin{aligned}
\operatorname{tr}(A) & =n-1+a_{s, s} \\
& =n-1+a_{1,1} \cdots a_{s, s} \cdots a_{n, n} \\
& =n-1+\operatorname{per}(A) .
\end{aligned}
$$

Suppose there are exactly $m$ elements among $a_{1,1}, \ldots, a_{n, n}$ which are strictly less than 1 where $m \geq 2$. Without loss of generality, we can assume that $a_{1,1}<1, \ldots, a_{m, m}<1, a_{m+1, m+1}=\cdots=a_{n, n}=1$. Since $\left(1-a_{1,1}\right)\left(1-a_{2,2}\right)>0$, which implies $a_{1,1}+a_{2,2}<1+a_{1,1} a_{2,2}$, we have

$$
a_{1,1}+a_{2,2}+\cdots+a_{m, m}<1+a_{1,1} a_{2,2}+a_{3,3}+\cdots+a_{m, m} .
$$

Again since $a_{1,1} a_{2,2}+a_{3,3}<1+a_{1,1} a_{2,2} a_{3,3}$, we have

$$
1+a_{1,1} a_{2,2}+\cdots+a_{m, m}<2+a_{1,1} a_{2,2} a_{3,3}+\cdots+a_{m, m} .
$$

Eventually, we have

$$
a_{1,1}+a_{2,2}+\cdots+a_{m, m}<m-1+a_{1,1} \cdots a_{m, m} .
$$

Thus,

$$
\begin{aligned}
\operatorname{tr}(A) & =a_{1,1}+\cdots+a_{m, m}+\cdots+a_{n, n} \\
& <m-1+a_{1,1} \cdots a_{m, m}+n-m \\
& =n-1+a_{1,1} \cdots a_{m, m} \cdots a_{n, n} .
\end{aligned}
$$

Therefore, (2.3) holds for a diagonal matrix with equality if and only if at least $n-1$ elements in $a_{1,1}, \ldots, a_{n, n}$ are equal to 1.

Lemma 2.2. Let $A \geq 0$ a square matrix of order $n$ and $0 \leq a_{i, i} \leq 1$ for all $1 \leq i \leq n$. Then

$$
(n-1) \operatorname{tr}(A) \leq n(n-2)+\operatorname{per}\left(A_{1,1}\right)+\cdots+\operatorname{per}\left(A_{n, n}\right) .
$$

If $A$ is a diagonal matrix, then the inequality holds with equality if and only if at least $n-1$ diagonal elements of $A$ are equal to 1 .

Proof. Applying Lemma 2.1 on $A_{i, i}$ for each $1 \leq i \leq n$, we have

$$
\operatorname{tr}\left(A_{i, i}\right)=\sum_{k \neq i} a_{k, k} \leq n-2+\operatorname{per}\left(A_{i, i}\right) .
$$

Summing all the inequalities over $i$ from 1 to $n$, we get (2.8).

Lemma 2.3. Let $A \geq 0$. Then

$$
\sum_{i=1}^{n} r_{i} \operatorname{per}\left(A_{i, i}\right) \leq r_{1} r_{2} \cdots r_{n}+(n-1) \operatorname{per}(A)
$$

where $r_{i}$ denote the $i$ th row sum of $A$ for $1 \leq i \leq n$. 
Proof. Since $\sum_{j=1}^{n} a_{i, j}=r_{i}, 1 \leq i \leq n$, we have

$$
\sum_{1 \leq j_{1}, \ldots, j_{n} \leq n} a_{1, j_{1}} a_{2, j_{2}} \cdots a_{n, j_{n}}=r_{1} r_{2} \cdots r_{n}
$$

Moreover, we have

$$
\begin{aligned}
r_{1} r_{2} \cdots r_{n} \geq & \operatorname{per}(A)+\left(a_{1,2}+\cdots+a_{1, n}\right) \operatorname{per}\left(A_{1,1}\right) \\
& +\left(a_{2,1}+a_{2,3}+\cdots+a_{2, n}\right) \operatorname{per}\left(A_{2,2}\right)+\cdots \\
& +\left(a_{n, 1}+a_{n, 2}+\cdots+a_{n, n-1}\right) \operatorname{per}\left(A_{n, n}\right) \\
= & \operatorname{per}(A)+\sum_{i=1}^{n}\left(r_{i}-a_{i, i}\right) \operatorname{per}\left(A_{i, i}\right) .
\end{aligned}
$$

Combine with $a_{i, i} \operatorname{per}\left(A_{i, i}\right) \leq \operatorname{per}(A)$ for all $1 \leq i \leq n$, we prove the lemma.

The inequality in Lemma 2.3 is strict if $A>0$. From the proof of Lemma 2.3, if $A$ is row stochastic, then by inequality (2.9) we have the following corollary.

Corollary 2.4. Let $A$ be an $n \times n$ row stochastic matrix. Then

$$
\sum_{i=1}^{n}\left(1-a_{i, i}\right) \operatorname{per}\left(A_{i, i}\right) \leq 1-\operatorname{per}(A) .
$$

Lemma 2.5. Let $A$ be an $n \times n$ row substochastic matrix. Then

$$
\sum_{i=1}^{n}\left(1-r_{i}\right) \operatorname{per}\left(A_{i, i}\right) \leq 1-r_{1} r_{2} \cdots r_{n}
$$

The inequality holds with equality if and only if either $A$ is a diagonal matrix with at least $(n-1)$ diagonal elements equal to 1 , or the $k$ th row and the $k$ th column of $A$ are all zero's for some $1 \leq k \leq n$ and $A_{k, k}$ is a permutation matrix.

Proof. Since

$$
\operatorname{per}\left(A_{i, i}\right) \leq \prod_{s \neq i} r_{s}
$$

we have

$$
\sum_{i=1}^{n}\left(1-r_{i}\right) \operatorname{per}\left(A_{i, i}\right) \leq \sum_{i=1}^{n}\left(1-r_{i}\right) \prod_{s \neq i} r_{s}
$$

So we only need to show

$$
\sum_{i=1}^{n}\left(1-r_{i}\right) \prod_{s \neq i} r_{s} \leq 1-r_{1} r_{2} \cdots r_{n} .
$$

We use induction to prove it. For $n=2$, since $\left(1-r_{1}\right)\left(1-r_{2}\right) \geq 0$, we have

$$
\left(1-r_{1}\right) r_{2}+\left(1-r_{2}\right) r_{1} \leq 1-r_{1} r_{2} .
$$


Suppose the inequality (2.13) holds when $n=k$. For $n=k+1$, we have

$$
\begin{aligned}
\sum_{i=1}^{k+1}\left(\left(1-r_{i}\right) \prod_{s \neq i} r_{s}\right) & =\left(\sum_{i=1}^{k}\left(1-r_{i}\right) \prod_{\substack{s \neq i \\
1 \leq s \leq k}} r_{s}\right) r_{k+1}+\left(1-r_{k+1}\right) \prod_{s \neq k+1} r_{s} \\
& \leq\left(1-r_{1} r_{2} \cdots r_{k}\right) r_{k+1}+r_{1} r_{2} \cdots r_{k}-r_{1} r_{2} \cdots r_{k} r_{k+1} \\
& =1-r_{1} r_{2} \cdots r_{k+1}+\left(1-r_{1} r_{2} \cdots r_{k}\right)\left(r_{k+1}-1\right) \\
& \leq 1-r_{1} r_{2} \cdots r_{k+1} .
\end{aligned}
$$

Inequality (2.12) holds with equality if and only if (2.11) holds with equality for all $1 \leq i \leq n$. If $A$ has at least two row sums equal to zero, then $\operatorname{per}\left(A_{i, i}\right)=\prod_{s \neq i} r_{s}=0$. If $A$ has exactly one row sum $r_{k}=0$, then for $i \neq k, \operatorname{per}\left(A_{i, i}\right)=\prod_{s \neq i} r_{s}=0 . \operatorname{per}\left(A_{k, k}\right)=\prod_{s \neq k} r_{s}$ if and only if the $k$ th column of $A$ are all zero's and $A_{k, k}$ has exactly one positive element on each row and each column. If all row sums of $A$ are positive, (2.11) holds with equality for all $1 \leq i \leq n$ if and only if $A$ is a diagonal matrix with $a_{i, i}>0$ for all $1 \leq i \leq n$. Thus, (2.12) holds with equality if and only if $A$ satisfies any one of the following conditions.

(i) $A$ has at least two row sums equal to zero.

(ii) There exists some $1 \leq k \leq n$ such that the $k$ th row and the $k$ th column of $A$ are all zero's, and $A_{k, k}$ contains exactly one positive element on each row and each column.

(iii) $A$ is a diagonal matrix with $a_{i, i}>0$ for all $1 \leq i \leq n$.

We then show that (2.13) holds with equality if and only if at least $n-1$ row sums of $A$ are 1 . It is easy to check that if $A$ has at least $n-1$ row sums equal to 1 , then (2.13) holds with equality. Suppose there are exactly $m$ row sums of $A$ strictly less than 1 where $m \geq 2$. Without loss of generality, we can assume that $r_{1}<1, \ldots, r_{m}<1, r_{m+1}=\cdots=r_{n}=1$. Then

$$
\begin{aligned}
\sum_{i=1}^{n}\left(1-r_{i}\right) \prod_{s \neq i} r_{s} & =\sum_{i=1}^{m}\left(1-r_{i}\right) \prod_{s \neq i} r_{s} \\
& <\left(1-r_{1} r_{2}\right) r_{3} \cdots r_{m}+r_{1} r_{2}\left(1-r_{3}\right) \cdots r_{m}+\cdots+r_{1} \cdots r_{m-1}\left(1-r_{m}\right) \\
& <\left(1-r_{1} r_{2} r_{3}\right) r_{4} \cdots r_{m}+r_{1} r_{2} r_{3}\left(1-r_{4}\right) \cdots r_{m}+\cdots+r_{1} \cdots r_{m-1}\left(1-r_{m}\right) \\
& <\cdots<1-r_{1} r_{2} \cdots r_{m}=1-r_{1} r_{2} \cdots r_{n} .
\end{aligned}
$$

Since (2.10) holds with equality if and only if (2.12) and (2.13) holds with equality, the lemma is proved.

The following corollary follows by Lemmas 2.3 and 2.5 .

Corollary 2.6. Let $A$ be an $n \times n$ row substochastic matrix. Then

$$
\sum_{i=1}^{n} \operatorname{per}\left(A_{i, i}\right) \leq 1+(n-1) \operatorname{per}(A) .
$$

The inequality holds with equality if and only if either $A$ is a diagonal matrix with at least $(n-1)$ diagonal elements equal to 1 , or the $k$ th row and the $k$ th column of $A$ are all zero's for some $1 \leq k \leq n$ and $A_{k, k}$ is a permutation matrix.

The inequality in (2.14) is strict if $A>0$. 
Lemma 2.7. Given $A^{\prime}=\left[a_{i, j}^{\prime}\right] \geq 0, A=\left[a_{i, j}\right] \geq 0$ satisfying that $A^{\prime}-A \geq 0$ and $0 \leq a_{i, i}^{\prime}=a_{i, i} \leq 1$ for all $1 \leq i \leq n$, then

$$
\operatorname{per}\left(A^{\prime}\right)+\sum_{i=1}^{n}\left(1-a_{i, i}^{\prime}\right) \operatorname{per}\left(A_{i, i}^{\prime}\right) \geq \operatorname{per}(A)+\sum_{i=1}^{n}\left(1-a_{i, i}\right) \operatorname{per}\left(A_{i, i}\right) .
$$

Proof. Since $A^{\prime}, A \geq 0$ and $A^{\prime}-A \geq 0, \operatorname{per}\left(A^{\prime}\right) \geq \operatorname{per}(A)$ and $\operatorname{per}\left(A_{i, i}^{\prime}\right) \geq \operatorname{per}\left(A_{i, i}\right)$ for $1 \leq i \leq n$. Also since $a_{i, i}^{\prime}=a_{i, i}$, which implies $1-a_{i, i}^{\prime}=1-a_{i, i}$ for $1 \leq i \leq n$, thus the inequality (2.15) holds.

Lemma 2.8. Let $A=\left[a_{i, j}\right] \geq 0$ satisfying $0 \leq a_{i, i} \leq 1$ for all $1 \leq i \leq n$, and $A^{(\epsilon, k)}=\left[a_{i, j}^{\prime}\right]$ for some $1 \leq k \leq n$ and $\epsilon \geq 0$, where

$$
a_{i, j}^{\prime}=\left\{\begin{array}{ll}
a_{i, j} & \text { if }(i, j) \neq(k, k) \\
a_{k, k}+\epsilon & \text { if }(i, j)=(k, k)
\end{array} .\right.
$$

Then

$$
\operatorname{per}\left(A^{(\epsilon, k)}\right)+\sum_{i=1}^{n}\left(1-a_{i, i}^{\prime}\right) \operatorname{per}\left(A_{i, i}^{(\epsilon, k)}\right) \geq \operatorname{per}(A)+\sum_{i=1}^{n}\left(1-a_{i, i}\right) \operatorname{per}\left(A_{i, i}\right)
$$

Proof. Note that $A_{k, k}^{(\epsilon, k)}=A_{k, k}$, we have

$$
\begin{aligned}
& \operatorname{per}\left(A^{(\epsilon, k)}\right)+\sum_{i=1}^{n}\left(1-a_{i, i}^{\prime}\right) \operatorname{per}\left(A_{i, i}^{(\epsilon, k)}\right) \\
= & \operatorname{per}(A)+\epsilon \operatorname{per}\left(A_{k, k}\right)+\left(1-a_{1,1}\right) \operatorname{per}\left(A_{1,1}^{(\epsilon, k)}\right)+\cdots \\
& +\left(1-a_{k, k}-\epsilon\right) \operatorname{per}\left(A_{k, k}\right)+\cdots+\left(1-a_{n, n}\right) \operatorname{per}\left(A_{n, n}^{(\epsilon, k)}\right) \\
= & \operatorname{per}(A)+\sum_{i=1}^{n}\left(1-a_{i, i}\right) \operatorname{per}\left(A_{i, i}^{(\epsilon, k)}\right) \\
\geq & \operatorname{per}(A)+\sum_{i=1}^{n}\left(1-a_{i, i}\right) \operatorname{per}\left(A_{i, i}\right) .
\end{aligned}
$$

Lemma 2.7 and Lemma 2.8 imply the following corollary.

Corollary 2.9. Let $A=\left[a_{i, j}\right] \geq 0, B=\left[b_{i, j}\right] \geq 0, A-B \leq 0$ and $0 \leq a_{i, i} \leq b_{i, i} \leq 1$. Then

$$
\operatorname{per}(A)+\sum_{i=1}^{n}\left(1-a_{i, i}\right) \operatorname{per}\left(A_{i, i}\right) \leq \operatorname{per}(B)+\sum_{i=1}^{n}\left(1-b_{i, i}\right) \operatorname{per}\left(B_{i, i}\right)
$$

Lemma 2.10. Let $A$ be an $n \times n$ row substochastic matrix. Then there exists a row stochastic matrix $B$ such that $A \leq B$.

Proof. For each $1 \leq i \leq n$, we can add $1-r_{i}$ on any entry in the $i$ th row of $A$, where $r_{i}$ denotes the $i$ th row sum of $A$. The resultant matrix is row stochastic.

Lemma 1.1 can then be generalized to the row substochastic matrix case.

Theorem 2.11. Let $A$ be an $n \times n$ row substochastic matrix. Then

$$
\sum_{i=1}^{n}\left(1-a_{i, i}\right) \operatorname{per}\left(A_{i, i}\right) \leq 1-\operatorname{per}(A) .
$$


Proof. By Lemma 2.10, we can find a row stochastic matrix $B$ such that $A \leq B$. According to Corollary 2.9 , we have

$$
\operatorname{per}(A)+\sum_{i=1}^{n}\left(1-a_{i, i}\right) \operatorname{per}\left(A_{i, i}\right) \leq \operatorname{per}(B)+\sum_{i=1}^{n}\left(1-b_{i, i}\right) \operatorname{per}\left(B_{i, i}\right)
$$

Applying Corollary 2.4, we have

$$
\operatorname{per}(B)+\sum_{i=1}^{n}\left(1-b_{i, i}\right) \operatorname{per}\left(B_{i, i}\right) \leq 1
$$

Thus, (2.16) holds.

REMARK 2.12. In Corollary 2.6, (2.14) can be obtained by adding (2.16) and the following inequality

$$
\sum_{i=1}^{n} a_{i, i} \operatorname{per}\left(A_{i, i}\right) \leq n \cdot \operatorname{per}(A) .
$$

The following lemma was proved by Brualdi and Newman [2].

Lemma 2.13. [2, Lemma 1] Let $C$ be a non-empty convex subset of vector space. Let $f$ be a real-valued function defined over $C$. Let $x$ be a fixed element of $C$. Then if there is an $\epsilon$ such that $0<\epsilon \leq 1$ and the inequality

$$
f(\alpha x+(1-\alpha) y) \leq \alpha f(x)+(1-\alpha) f(y),
$$

holds for all $\alpha$ in $[0, \epsilon]$ and all $y$ in $C$, then (2.17) also holds for all $\alpha$ in $[0,1]$ and all $y$ in $C$.

Now we are ready to prove the convex property of permanent between identity matrix $I_{n}$ and row substochastic matrices of order $n$.

TheOREM 2.14. Let $A$ be an $n \times n$ row substochastic matrix. Then

$$
\operatorname{per}\left(\alpha I_{n}+(1-\alpha) A\right) \leq \alpha+(1-\alpha) \operatorname{per}(A)
$$

for $0 \leq \alpha \leq 1$.

Proof. It is easy to check that (2.18) holds under the cases that either $A$ is a diagonal matrix with at least $(n-1)$ diagonal elements equal to 1 , or the $k$ th row and the $k$ th column of $A$ are all zero's for some $1 \leq k \leq n$ and $A_{k, k}$ is a permutation matrix. So we just need to consider the situation when $A$ is not in these two cases. The following identity

$$
\operatorname{per}\left(\alpha I_{n}+(1-\alpha) A\right)=\sum_{k=0}^{n}(1-\alpha)^{n-k} \alpha^{k} E_{n-k}(A)
$$

holds, where $E_{k}(A)$ is the sum of all $\left(\begin{array}{l}n \\ k\end{array}\right)$ principal $k \times k$ permanental minors of $A$. Since

$$
\operatorname{per}\left(\alpha I_{n}+(1-\alpha) A\right)=\operatorname{per}(A)+\left\{E_{n-1}(A)-n \cdot \operatorname{per}(A)\right\} \alpha+O\left(\alpha^{2}\right),
$$


by Corollary 2.6, we have

$$
\begin{aligned}
& \alpha+(1-\alpha) \operatorname{per}(A)-\operatorname{per}\left(\alpha I_{n}+(1-\alpha) A\right) \\
= & {\left[1+(n-1) \operatorname{per}(A)-E_{n-1}(A)\right] \alpha+O\left(\alpha^{2}\right) } \\
= & {\left[1+(n-1) \operatorname{per}(A)-\sum_{i=1}^{n} \operatorname{per}\left(A_{i, i}\right)\right] \alpha+O\left(\alpha^{2}\right) \geq 0, }
\end{aligned}
$$

for all sufficiently small non-negative $\alpha$ because the linear term in $\alpha$ is strictly greater than 0 . Since the set of all row substochastic matrices is a closed convex polytope, the smallest positive root of the polynomial equation $\operatorname{per}\left(\alpha I_{n}+(1-\alpha) A\right)-\alpha-(1-\alpha) \operatorname{per}(A)=0$, viewed as a function of $A$, is continuous. Thus, it has minimum value on the set of all row substochastic matrices, which is positive. This implies that (2.18) holds for all sufficiently small non-negative $\alpha$. By Lemma 2.13, the theorem holds.

REMARK 2.15. Since every doubly stochastic matrix is a row substochastic matrix, Theorem 1.2 by Brualdi and Newman is implied by Theorem 2.14. By the way, every doubly substochastic matrix is row substochastic, Theorem 2.14 also holds for all matrices in the convex polytope $\omega_{n}$.

3. A conjecture. For any nonnegative real vector $R=\left(r_{1}, r_{2}, \ldots, r_{n}\right)$, denote the rearrangement of the elements in $R$ in ascending order by

$$
r_{1}^{\prime} \leq r_{2}^{\prime} \leq \cdots \leq r_{n}^{\prime}
$$

and we denote

$$
R^{\prime}=\left(r_{1}^{\prime}, r_{2}^{\prime}, \ldots, r_{n}^{\prime}\right) .
$$

Similarly denote by $S^{\prime}$ the rearrangement of the elements in $S$ in ascending order.

Given $R$ and $S$ satisfying the compatible condition (1.1) with $m=n$, we denote $\mathfrak{E}(R, S)$ the set of all extreme points of $\mathcal{U}(R, S)$. The following theorem is given by Jurkat and Ryser [6].

Theorem 3.1. [6] Let $A$ be a matrix in $\mathcal{U}(R, S)$, then

$$
\operatorname{per}(A) \leq \prod_{i=1}^{n} \min \left\{r_{i}^{\prime}, s_{i}^{\prime}\right\}
$$

and equality is attained in (3.19) by a matrix $A$ in $\mathfrak{E}\left(R^{\prime}, S^{\prime}\right)$ with main diagonal

$$
a_{i, i}=\min \left\{r_{i}^{\prime}, s_{i}^{\prime}\right\}, \quad \text { for all } 1 \leq i \leq n .
$$

For $A=\left[a_{i, j}\right]$ an $n \times n$ matrix, define

$$
f(A)=1-\operatorname{per}(A)-\sum_{i=1}^{n}\left(1-a_{i, i}\right) \operatorname{per}\left(A_{i, i}\right) .
$$

According to Theorem 2.11, if $A$ is row substochastic, then $f(A) \geq 0$. The following lemma can be verified by a direct computation using Laplace expansion. 
Lemma 3.2. Let $R=\left(r_{1}, \ldots, r_{n}\right)$ and $S=\left(s_{1}, \ldots, s_{n}\right)$ satisfying condition (1.1) with $0 \leq r_{i} \leq 1$ for all $1 \leq i \leq n$. Suppose that $D=\left[d_{i, j}\right]$ and $E=\left[e_{i, j}\right]$ in $\mathfrak{E}(R, S)$ with

$$
d_{i, i}=e_{i, i}=\min \left\{r_{i}, s_{i}\right\}, \quad \text { for all } 1 \leq i \leq n .
$$

Then

$$
f(D)=f(E) \geq 0 .
$$

LEMmA 3.3. Let $U=\left(u_{1}, \ldots, u_{n}\right)$ and $V=\left(v_{1}, \ldots, v_{n}\right)$ be two $n$ dimensional nonnegative vectors satisfying $0 \leq u_{i} \leq v_{i} \leq 1$ for all $1 \leq i \leq n$. Then we have

$$
\prod_{i=1}^{n} u_{i}+\sum_{i=1}^{n}\left(1-u_{i}\right) \prod_{\substack{j=1 \\ j \neq i}}^{n} u_{j} \leq \prod_{i=1}^{n} v_{i}+\sum_{i=1}^{n}\left(1-v_{i}\right) \prod_{\substack{j=1 \\ j \neq i}}^{n} v_{j} .
$$

Proof. We prove the lemma by induction. When $n=2$, we have

$$
\begin{aligned}
& u_{1} u_{2}+\left(1-u_{1}\right) u_{2}+u_{1}\left(1-u_{2}\right)=1-\left(1-u_{1}\right)\left(1-u_{2}\right) \\
& \leq 1-\left(1-v_{1}\right)\left(1-v_{2}\right)=v_{1} v_{2}+\left(1-v_{1}\right) v_{2}+v_{1}\left(1-v_{2}\right) .
\end{aligned}
$$

Suppose (3.20) holds for $n=k$, then for $n=k+1$,

$$
\begin{aligned}
& u_{1} \cdots u_{k} u_{k+1}+\sum_{i=1}^{k+1}\left(1-u_{i}\right) \prod_{\substack{j=1 \\
j \neq i}}^{k+1} u_{j} \\
= & {\left[u_{1} \cdots u_{k}+\sum_{i=1}^{k}\left(1-u_{i}\right) \prod_{\substack{j=1 \\
j \neq i}}^{k} u_{j}\right] u_{k+1}+\left(1-u_{k+1}\right) u_{1} \cdots u_{k} } \\
\leq & {\left[v_{1} \cdots v_{k}+\sum_{i=1}^{k}\left(1-v_{i}\right) \prod_{\substack{j=1 \\
j \neq i}}^{k} v_{j}\right] u_{k+1}+\left(1-u_{k+1}\right) v_{1} \cdots v_{k} } \\
\leq & v_{1} \cdots v_{k} v_{k+1}+\sum_{i=1}^{k}\left(1-v_{i}\right) \prod_{\substack{j=1 \\
j \neq i}}^{k} v_{j} v_{k+1}+\left(1-v_{k+1}\right) v_{1} \cdots v_{k} \\
= & v_{1} \cdots v_{k} v_{k+1}+\sum_{i=1}^{k+1}\left(1-v_{i}\right) \prod_{\substack{j=1 \\
j \neq i}}^{k+1} v_{j} .
\end{aligned}
$$

TheOREM 3.4. Let $R=\left(r_{1}, \ldots, r_{n}\right)$ and $S=\left(s_{1}, \ldots, s_{n}\right)$ satisfying condition (1.1). If $0 \leq r_{i} \leq 1$ for all $1 \leq i \leq n$, then

$$
f(E) \geq f(\tilde{E}) \geq 0
$$

where $E \in \mathfrak{E}(R, S)$ has the main diagonal

$$
e_{i, i}=\min \left\{r_{i}, s_{i}\right\}, \quad \text { for all } 1 \leq i \leq n,
$$


and $\tilde{E} \in \mathfrak{E}\left(R^{\prime}, S^{\prime}\right)$ has the main diagonal

$$
\tilde{e}_{i, i}=\min \left\{r_{i}^{\prime}, s_{i}^{\prime}\right\}, \quad \text { for all } 1 \leq i \leq n,
$$

and $R^{\prime}=\left(r_{1}^{\prime}, \ldots, r_{n}^{\prime}\right), S^{\prime}=\left(s_{1}^{\prime}, \ldots, s_{n}^{\prime}\right)$.

Proof. Let $\operatorname{diag} E=\left(e_{1,1}, \ldots, e_{n, n}\right)$, where $e_{i, i}=\min \left\{r_{i}, s_{i}\right\}$ for all $1 \leq i \leq n$. By rearranging the elements in $\operatorname{diag} E$ in ascending order, we have $(\operatorname{diag} E)^{\prime}=\left(e_{1,1}^{\prime}, \ldots, e_{n, n}^{\prime}\right)$, where $e_{1,1}^{\prime} \leq \cdots \leq e_{n, n}^{\prime}$. Since $0 \leq e_{i, i}^{\prime} \leq \tilde{e}_{i, i} \leq 1$, by Lemma 3.3, we have

$$
\begin{aligned}
f(E) & =1-e_{1,1} \cdots e_{n, n}-\sum_{i=1}^{n}\left(1-e_{i, i}\right) \prod_{\substack{j=1 \\
j \neq i}}^{n} e_{j, j} \\
& =1-e_{1,1}^{\prime} \cdots e_{n, n}^{\prime}-\sum_{i=1}^{n}\left(1-e_{i, i}^{\prime}\right) \prod_{\substack{j=1 \\
j \neq i}}^{n} e_{j, j}^{\prime} \\
& \geq 1-\tilde{e}_{1,1} \cdots \tilde{e}_{n, n}-\sum_{i=1}^{n}\left(1-\tilde{e}_{i, i}\right) \prod_{\substack{j=1 \\
j \neq i}}^{n} \tilde{e}_{j, j}=f(\tilde{E}) .
\end{aligned}
$$

Thus, the theorem holds.

REMARK 3.5. When $R=S=(1, \ldots, 1)$, by taking $E=\left[e_{i, j}\right]=I_{n}$ where $e_{i, i}=\min \left\{r_{i}, s_{i}\right\}=1$ for all $0 \leq i \leq n$, we have $f\left(I_{n}\right)=0$ which gives the minimum value of $f$ on $\Omega_{n}$. However this might not be true for the general case. For example, let $R=(0.07,0.25,0.23,0.25)$ and $S=(0.51,0.05,0.1,0.14)$. If

$$
E=\left[\begin{array}{cccc}
0.07 & 0 & 0 & 0 \\
0.2 & 0.05 & 0 & 0 \\
0.13 & 0 & 0.1 & 0 \\
0.11 & 0 & 0 & 0.14
\end{array}\right]
$$

such that $e_{i, i}=\min \left\{r_{i}, s_{i}\right\}$ for $1 \leq i \leq 4$, and

$$
\hat{E}=\left[\begin{array}{cccc}
0.02 & 0.05 & 0 & 0 \\
0.25 & 0 & 0 & 0 \\
0.13 & 0 & 0.1 & 0 \\
0.11 & 0 & 0 & 0.14
\end{array}\right]
$$

then we get $f(E) \approx 0.9976269$ and $f(\hat{E})=0.996895$.

Remark 3.6. Let $R=S=(1, \ldots, 1)$. If $P$ is an extreme point of $\mathcal{U}(R, S)=\Omega_{n}$, meaning that $P$ is a permutation matrix, then $f(P)=0$. But $f(A)=0$ is not sufficient for $A$ being an extreme point. For example, if

$$
A=\left[\begin{array}{cccc}
1 & \cdots & 0 & 0 \\
\vdots & \ddots & \vdots & \vdots \\
0 & \cdots & 0.5 & 0.5 \\
0 & \cdots & 0.5 & 0.5
\end{array}\right] \in \Omega_{n}
$$

$f(A)=0$, but $A$ is not an extreme point. 
ConjeCture 3.7. Let $R=\left(r_{1}, \ldots, r_{n}\right)$ and $S=\left(s_{1}, \ldots, s_{n}\right)$ satisfying condition (1.1), where $0 \leq r_{i} \leq 1$ for all $1 \leq i \leq n$. Then

$$
\min \{f(A) \mid A \in \mathcal{U}(R, S)\}=\min \{f(E \mid E \in \mathfrak{E}(R, S)\}) .
$$

EXAMPLE 3.8. Let $R=(0.51,0.99,0.95,0.99)$ and $S=(2.98,0.3,0.05,0.11)$. Let

$$
A=\left[\begin{array}{cccc}
0.35 & 0.04 & 0.02 & 0.1 \\
0.72 & 0.23 & 0.03 & 0.01 \\
0.94 & 0.01 & 0 & 0 \\
0.97 & 0.02 & 0 & 0
\end{array}\right]
$$

and

$$
E=\left[\begin{array}{cccc}
0.21 & 0.3 & 0 & 0 \\
0.99 & 0 & 0 & 0 \\
0.9 & 0 & 0.05 & 0 \\
0.88 & 0 & 0 & 0.11
\end{array}\right]
$$

By direct computations, we have $f(A)=0.9699998$ and $f(E)=0.9529585$, which satisfy $f(A)>f(E)$.

Acknowledgment. We are very grateful to an anonymous referee for his/her valuable suggestions and comments, which help us to improve the results of this paper.

\section{REFERENCES}

[1] G. Birkhoff. Tres observaciones sobre el algebra lineal. Univ. Nac. Tucumán, Revista, Ser. A, 5:147-151, 1946.

[2] R.A. Brualdi and M. Newman. Inequalities for permanents and permanental minors. Math. Proc. Cambridge Philos. Soc., 61:741-746, 1965.

[3] L. Cao, Z. Chen, X. Duan, S. Koyuncu, and H. Li. Diagonal sums of doubly substochastic matrices. Electron. J. Linear Algebra, 35:42-52, 2019

[4] L. Cao, Z. Chen, S. Koyuncu, and H. Li. Permanents of doubly substochastic matrices. Linear Multilinear Algebra, doi:10.1080/03081087.2018.1513448, 2018.

[5] Z. Chen and L. Cao On the maximum of the permanent of $(I-A)$. Linear Algebra Appl., 555:412-431, 2018.

[6] W.B. Jurkat and H.J. Ryser. Term ranks and permanents of nonnegative matrices. J. Algebra, 5(3):342-357, 1967.

[7] L. Mirsky. On a convex set of matrices. Arch. Math., 10(1):88-92, 1959. 